\title{
New Hepatitis C Virus (HCV) Drugs and the Hope for a Cure: Concepts in Anti-HCV Drug Development
}

\author{
Jean-Michel Pawlotsky, MD, $\mathrm{PhD}^{1,2}$ \\ ${ }^{1}$ National Reference Center for Viral Hepatitis B, C and D, Department \\ of Virology, Hôpital Henri Mondor, Université Paris-Est, Créteil, \\ France \\ 2 INSERM U955, Créteil, France
}

\begin{abstract}
Address for correspondence Jean-Michel Pawlotsky, MD, PhD, Department of Virology, Hôpital Henri Mondor, 51 avenue du Maréchal de Lattre de Tassigny, 94010 Créteil, France

(e-mail: jean-michel.pawlotsky@hmn.aphp.fr).
\end{abstract}

\begin{abstract}
Keywords

- hepatitis C virus

- direct-acting antivirals

- host-targeted agents

- simeprevir

- sofosbuvir

The development of new models and tools has led to the discovery and clinical development of a large number of new anti-hepatitis $\mathrm{C}$ virus (HCV) drugs, including direct-acting antivirals and host-targeted agents. Surprisingly, curing HCV infection appears to be easy with these new drugs, provided that a potent drug combination with a high barrier to resistance is used. HCV infection cure rates can be optimized by combining drugs with synergistic antiviral effects, tailoring treatment duration to the patients' needs, and/or using ribavirin. Two HCV drugs have been approved in 2011telaprevir and boceprevir, both first-wave, first-generation NS3-4A protease inhibitors, two others in 2013/2014-simeprevir, a second-wave, first-generation NS3-4A protease inhibitor, and sofosbuvir, a nucleotide analogue inhibitor of the viral polymerase. Numerous other drugs have reached phase II or III clinical development. From 2015 and onwards, interferon-containing regimens will disappear, replaced by interferon-free regimens yielding infection cure rates over $90 \%$. These therapies will raise new issues, including the need for broad-scale screening and access to care.
\end{abstract}

When hepatitis $\mathrm{C}$ virus (HCV) was discovered in the late $1980{ }^{1}{ }^{1}$ the only available treatment for what was known as chronic "non-A, non-B" hepatitis was standard interferonalpha (IFN- $\alpha$ ), 3 million units three times per week subcutaneously for 24 weeks. Only $6 \%$ of patients achieved a sustained virological response (SVR), i.e. a cure of HCV infection, with this regimen. The initial choice of IFN- $\alpha$ as a potential treatment for chronic hepatitis $C$ was an empirical one. In 1986, Hoofnagle et $\mathrm{al}^{2}$ had written: "Alpha interferon was a natural choice as a possible therapeutic agent for chronic nonA, non-B hepatitis. This agent has a wide spectrum of antiviral activity and has been used to treat many acute and chronic viral illnesses. Alpha interferon has already been shown to inhibit replication of several human hepatitis viruses, including hepatitis A virus (in cell culture), hepatitis B virus and the hepatitis delta agent." Prolonging IFN- $\alpha$ administration to
48 weeks increased the SVR rate up to 12 to $16 \%$. Ribavirin was introduced in $1991^{3}$ with the following justification: "Ribavirin is a noninterferon-inducing nucleotide analogue with a broad spectrum of activity against RNA and DNA viruses, including those from the flavivirus family." In fact, ribavirin happened to be a very weak and transient direct inhibitor of HCV replication. ${ }^{4}$ However, when added to IFN- $\alpha$, ribavirin increased the SVR rates up to 40 to $45 \%$, through mechanisms that remain unknown. An additional 10\% increase in SVR rate was achieved when standard IFN- $\alpha$ was replaced by pegylated IFN- $\alpha$ PegIFN- $\alpha$ ), that could be administered once per week., ${ }^{5,6}$ Overall, $\sim 50 \%$ of HCV-infected patients cleared infection with the combination of PegIFN- $\alpha$ and ribavirin, with marked differences according to the HCV genotype. This treatment regimen remained the standard-of-care until 2011 for genotype 1, and until 2013 for the other genotypes. ${ }^{7}$
Issue Theme New Hepatitis C Therapies; Guest Editor, Jean-Michel Pawlotsky, MD, $\mathrm{PhD}$
Copyright $\odot 2014$ by Thieme Medical Publishers, Inc., 333 Seventh Avenue, New York, NY 10001, USA. Tel: +1(212) 584-4662.
DOI http://dx.doi.org/ 10.1055/s-0034-1371007. ISSN 0272-8087. 
The development of new models and tools used to unravel the different steps of the HCV lifecycle and an unprecedented effort of the academic field and drug industry toward the discovery and clinical development of new anti-HCV drugs led to the 2014 situation, with four approved new antiviral agents since 2011 and dozens at phase II or III clinical developmental stages. New HCV drugs split into two groups: direct-acting antivirals (DAAs) that target viral actors of the HCV lifecycle, and host-targeted agents (HTAs) that target host-cell components involved in complex interactions with viral proteins that are essential to the HCV lifecycle. The field quickly learned how to use these new compounds to optimize $\mathrm{HCV}$ infection cure rates. Here I describe the requirements for definitive cure of HCV, the HCV drugs currently in clinical development, and opportunities for new anti-HCV therapies in the short- to mid-term future.

\section{Curing HCV Infection: An Easy Task}

Surprisingly, curing HCV infection appeared to be an easy task, as soon as appropriate tools became available. Curing HCV replicons was easy with IFN- $\alpha$, with or without ribavirin, as a result of the sustained direct antiviral effect of IFN- $\alpha$ in cell culture. ${ }^{8}$ It was more difficult in clinical practice because several factors influenced the ability of infected patients to respond to IFN- $\alpha$ antiviral effects. It is only recently that the prominent role of a genetic predisposition to IFN responsiveness (related to the so-called IL28B genotype) was discovered. ${ }^{9}$ The HCV genotype, that influences the ability of the virus to be inhibited by IFN- $\alpha$ and of the infected cells to cure themselves, and the severity of liver disease (extensive fibrosis or cirrhosis), that alters the latter, were both identified as important additional determinants of the SVR in IFN$\alpha /$ ribavirin-based regimens. ${ }^{5,6}$ Thus, drugs with an antiviral effect not influenced by host or viral parameters, such as the DAAs or HTAs currently in development, were needed to achieve higher cure rates.

With such drugs, curing HCV infection becomes an easy task, at two conditions (-Fig. 1): (1) The antiviral effectiveness of the combination of drugs used must be high enough to efficiently block virus production by infected cells. This shutdown of virus production is responsible for the firstphase HCV RNA decline, observed within the first 1 to 3 days of therapy. ${ }^{10}$ (2) The inhibition of virus production must be sustained on treatment. This implies that the combination of drugs has a high barrier to resistance that prevents resistant viruses from being selected and inducing a virological breakthrough in patients who are adherent to therapy. If both conditions are fulfilled, the progressive decrease of the number of infected cells is responsible for the observed second-phase decline of HCV RNA levels. It is the combined result of the natural death rate of infected cells and the rate of loss of the ability of the remaining infected cells to produce virus as their intracellular RNA degrades, which is the infected cell cure rate. ${ }^{11}$ The progressive loss of infected cells leads to a definitive cure of infection if the patient is still on treatment when the last infected cell is cleared or cured. Conversely, if the patient's body still contains infected cells

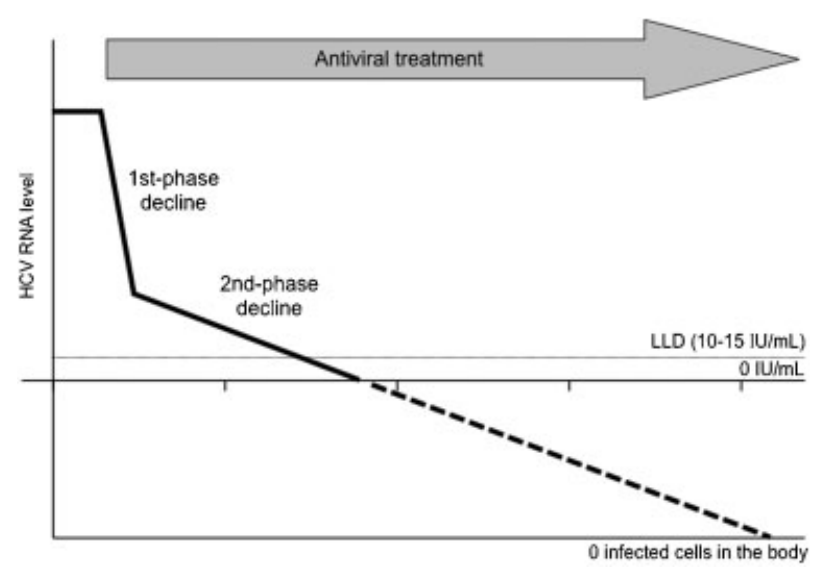

Fig. 1 Biphasic decline of HCV RNA levels on antiviral therapy leading to a cure of infection. The first-phase HCV RNA decline is the result of the direct antiviral effect of the drug combination used. No virological breakthrough occurs when a drug combination with a high barrier to resistance is used. As a result, the second-phase HCV RNA decline reflects the progressive clearance (cell death and cure) of infected cells, that initially leads to an undetectable HCV RNA in blood and continues below detection for weeks to months, according to the second-phase slope, until no more infected cells are present in the patient's body (cure of infection that translates into a sustained virological response). LLD, lower limit of detection of the HCV RNA assay.

when treatment is stopped, infection relapses within a few days to weeks after treatment cessation.

\section{Requirements for an HCV Cure}

\section{Antiviral Potency (First-Phase HCV RNA Decline)}

As discussed by Rupp and Bartenschlager in this issue of Seminars, ${ }^{12}$ the HCV lifecycle offers several potential targets for DAAs and HTAs with potent antiviral activity. The lifecycle starts with receptor binding, entry into cells, and fusion. Decapsidation of viral nucleocapsids liberates free positivestrand genomic RNAs in the cell cytoplasm, where they serve, together with newly synthesized RNAs, as messenger RNAs for synthesis of the HCV polyprotein. The polyprotein is then cleaved by host cell peptidases, the NS2 autocatalytic protease and the NS3-4A serine protease to generate three structural and seven nonstructural mature viral proteins. Replication is catalyzed by the HCV RNA-dependent RNA polymerase (RdRp), and the NS5A protein plays an important regulatory role in virus replication. HCV then uses the lipoprotein production pathway to generate mature viral particles and export them. ${ }^{12}$

Thus far, drugs from all classes of DAAs in clinical development, including NS3-4A protease inhibitors, nucleoside/ nucleotide analogue inhibitors of HCV RdRp, nonnucleoside inhibitors of RdRp, and NS5A inhibitors, have been shown to reduce $\mathrm{HCV}$ replication by 3 logs or more over 3 days of administration. HTAs, such as cyclophilin A inhibitors and the microRNA-122 (miR-122) antagonist, can also reduce viral replication by more than 3 logs within a week or 2 weeks. ${ }^{13,14}$ This antiviral effectiveness is sufficient to trigger the secondphase decline. Although this has been difficult to show in vivo, because most patients become HCV RNA undetectable early 
on treatment, combining drugs from different classes yields additive to synergistic antiviral effects, as suggested by in vitro studies. $^{15}$

\section{Barrier to Resistance (Sustainability of First-Phase HCV RNA Decline)}

Viral resistance corresponds to the selection during antiviral treatment of viral variants that bear amino acid substitutions altering the drug target, that are therefore less susceptible to the drug's inhibitory activity. ${ }^{16}$ These drug-resistant variants pre-exist, generally as minor populations, within the patient's quasispecies (the ensemble of all viral variant populations present in a given individual), as a result of the error-prone activity of the HCV RdRp, the large viral populations and the short half-life of the virus in peripheral blood. Drug exposure profoundly inhibits replication of the dominant, so-called wild-type, drug-sensitive viral population, and the resistant variants gradually occupy the vacant replication space. ${ }^{16}$

In vivo, viral resistance is influenced by three parameters that altogether, define the barrier to resistance of a drug or a drug class. They include (1) the genetic barrier to resistance, defined as the number of nucleotide/amino acid substitutions needed for a viral variant to acquire full resistance to the drug in question; (2) the in vivo fitness of the viral variant population, defined as its ability to survive and grow in the replicative environment; and (3) drug exposure, defined as the drug concentration achieved in vivo relative to the concentrations that must be achieved to efficiently inhibit replication of resistant variants. $^{16}$

HCV DAAS in development can be split into two groups. Drugs with a low barrier to resistance include first-generation NS3-4A protease inhibitors, first-generation nonnucleoside inhibitors of HCV RdRp and first-generation NS5A inhibitors. Nucleoside/nucleotide analogues have a high barrier to resistance because the resistant viral variants they select are poorly fit and therefore do not replicate at clinically meaningful levels when they are selected. Second-generation NS34A protease and NS5A inhibitors have a substantially higher barrier to resistance than first-generation drugs. However, they can select fit-resistant viruses, so there is room for thirdgeneration drugs with a truly high barrier to resistance. Finally, HTAs have a high barrier to resistance because they target conserved host-cell components involved in the HCV lifecycle, not variable viral proteins.

To ensure that the barrier to resistance of an antiviral treatment is high, drugs must be combined. Several options are available. They include (1) the combination of a drug with a high barrier to resistance (nucleoside/nucleotide analogue inhibitor of HCV RdRp or cyclophilin inhibitor), used as a "backbone," with one (or even two) other drugs with a low barrier to resistance; (2) the combination of three firstgeneration drugs with a low barrier to resistance (NS3-4A protease inhibitor, NS5A inhibitor, and nonnucleoside inhibitor of HCV RdRp). Two such drugs (NS3-4A protease inhibitor and NS5A inhibitor) may be sufficient in easy-to-cure patients, such as those infected with HCV genotype $1 \mathrm{~b}$ with a CC IL28B genotype; (3) the combination of two drugs including at least one second-generation NS3-4A protease or NS5A inhib- itor. These approaches have been assessed in phase II and III clinical trials and were all shown to ensure a high barrier to resistance in treatment-adherent patients.

\section{Clearance of Infected Cells (Second-Phase Decline)}

The loss/cure of infected cells upon antiviral therapy is under the control of multiple parameters, some of which can be modified to optimize treatment responses. The factors that cannot be modified include the patient's genetic background, disease characteristics such as extensive fibrosis or cirrhosis, and the HCV genotype. The factors that can be modulated include the antiviral effectiveness of the drug combination, treatment duration, and the use of ribavirin.

\section{Factors That Cannot Be Modified}

The IL28B genotype is a strong predictor of IFN responsiveness-the ability of IFN- $\alpha$ to significantly reduce HCV replication. ${ }^{9}$ In contrast, host genetics do not influence the firstphase decline on DAA-based therapy. However, the secondphase HCV RNA decline is influenced by the IL28B genotype in patients receiving IFN-free regimens. Indeed, the secondphase slope was shown to be significantly steeper in CC than in CT or TT patients. ${ }^{17,18}$ This means that higher SVR rates can be obtained with a given duration in CC patients, or alternatively, that longer treatment is needed in non-CC patients to achieve the same SVR rate with a given combination regimen. ${ }^{18}$ This observation also indicates that the ability of infected cells to be cured when virus production is blocked is genetically determined, and probably related to intracellular IFN responses, suggesting a key role of innate immunity in HCV clearance on therapy.

Extensive fibrosis and cirrhosis are associated with lower SVR rates on both IFN-containing and IFN-free combination regimens. ${ }^{19,20}$ This is due to a slower second-phase HCV RNA decline in cirrhotic than in noncirrhotic patients. The molecular mechanisms underlying the slower infected cell clearance in cirrhotic patients are unknown. It does not appear to be related to different drug exposures in cirrhotics because the first-phase response is identical in cirrhotic and noncirrhotic patients. Differences in the cirrhotic liver microenvironment might be responsible for subtle differences in the mechanisms of HCV clearance activated in infected cells on efficient antiviral therapy.

The HCV genotype was recently shown to influence the second-phase HCV RNA decline in patients receiving IFN-free regimens, through unknown mechanisms. Thus, with a given combination regimen, longer treatment is needed in patients infected with HCV genotypes that have a slower secondphase decline. ${ }^{19-21}$ Schematically, HCV genotypes can be classified as follows in order of slowing second-phase: genotype 2 , genotype $1 \mathrm{~b}$, genotype $1 \mathrm{a}$, and genotype 3 . For this reason, genotype 3 has become the most difficult-to-cure HCV genotype. Not enough data are available for genotypes 4 , 5 , and 6.

\section{Factors That Can Be Modified}

The loss of infected cells (second-phase slope) was shown to be significantly related to the antiviral effectiveness of the 
drug regimen used (first-phase slope). ${ }^{22}$ Thus, more potent antiviral drug combinations are associated with a more rapid loss of infected cells, even though the latter is not due to the direct antiviral effect of the drugs. It is therefore important to use potent, synergistic combinations of antiviral drugs to optimize both first-phase and second-phase HCV RNA declines.

Treatment duration is a key parameter that can be easily optimized to increase the SVR rates. Stopping treatment too early, that is before the last infected cell has been cleared or cured, results in reinfection of adjacent, then distant liver cells, and ultimately, in a virological relapse. In contrast, stopping therapy at any time after the last cell has been eliminated is associated with a definitive cure of infection. Thus, patients with a slow second-phase decline, such as patients with an unfavorable IL28B genotype, cirrhotics, patients infected with HCV genotype 3, etc., need longer therapy than those with a sharp one, regardless of the treatment regimen. As a result, one size does not fit all, and different subgroups of patients receiving a given drug combination may need different treatment durations to achieve an SVR, as recently shown with the combination of sofosbuvir and ribavirin in different groups of patients infected with HCV genotypes 1,2 , and $3 .^{19-21}$ This will also be true with more potent drug combinations that need shorter treatment durations. Thus, treatment duration with a given regimen must either be adapted to each subgroup of patients, or to simplify, tailored to the group that needs the longest treatment to avoid too many failures in the more difficult-to-cure patients. Current clinical development strategies aim to identify, for each treatment regimen, the shortest duration with the highest global SVR rate. This approach may be dangerous, as a treatment strategy validated in a phase III clinical trial may happen to be too short when applied to more difficult-to-cure real-life patients. The current race for shorter therapy may thus lead to undertreat a substantial proportion of patients in clinical practice.

Ribavirin accelerates the second-slope of viral decline in a dose-dependent manner in patients in whom virus production is efficiently blocked by IFN-containing or IFN-free drug combinations, through mechanisms that remain debated. ${ }^{4,23}$ Thus, ribavirin remains a useful tool to either reduce treatment duration or improve SVR rates with a given duration, as less time will be needed for all infected cells to be cleared/cured. Because ribavirin is cheap and relatively well tolerated when it is not combined with IFN- $\alpha$, it remains a very useful tool to fine tune anti-HCV treatment regimens and optimize their results.

\section{New Approved HCV Drugs or in Clinical Development}

\section{Approved Drugs}

Pegylated IFN- $\alpha 2 \mathrm{a}$ and IFN- $\alpha 2 \mathrm{~b}$ and ribavirin are still available for triple or quadruple IFN-containing regimens with new HCV drugs, as discussed by Aronsohn and Jensen in this issue. Telaprevir and boceprevir are first-wave, first-generation NS3-4A protease inhibitors that were approved in 2011 for use in combination with PegIFN- $\alpha$ and ribavirin. They are specific for genotype 1 and have a low barrier to resistance. Their use was associated with frequent side effects, including cutaneous complications, anemia, gastrointestinal disorders and renal toxicity for telaprevir; and anemia, dysgeusia, and renal toxicity for boceprevir, as well as with frequent drugdrug interactions due to their metabolism by cytochrome P450 CYP34A that they inhibit. ${ }^{24-29}$ For these reasons, these drugs will no longer be used when better tolerated compounds are available.

Two new HCV DAAs have been approved in the United States in December 2013, in Europe in the first half of 2014 simeprevir and sofosbuvir. Simeprevir is a second-wave, firstgeneration NS3-4A protease inhibitor. Its genotypic coverage is broader than that of the first-wave drugs, including at least genotypes 1,2 , and 4 ; however, simeprevir is inactive against HCV genotype 3. Simeprevir has a low barrier to resistance, with extensive cross resistance with telaprevir and boceprevir and the other first-generation NS3-4A protease inhibitors. In addition, simeprevir preferentially selects resistant variants bearing the Q80K substitution in the NS3 protease sequence. The presence of detectable levels of these variants at baseline has been associated with failures of simeprevircontaining regimens. ${ }^{30-32}$ Phase II and III clinical trials have shown an excellent tolerance profile for this compound. ${ }^{30-32}$ Simeprevir only modestly inhibits CYP34A.

Sofosbuvir is a first-generation uridine nucleotide analogue inhibitor of HCV RdRp, which is phosphorylated into its triphosphate form and incorporated into the RNA chain in formation, thus acting as a chain terminator. Sofosbuvir has pangenotypic antiviral activity, confirmed in vivo against genotypes 1 to $6 .^{19,20,33}$ In vitro, sofosbuvir selects variants with an S282T substitution in the RdRp sequence. ${ }^{33}$ However, these variants have considerably impaired replication capacity, both in vitro and in vivo. As a result, they have never been associated with virological breakthroughs on treatment, and were exceptionally found in patients who relapsed after treatment withdrawal. ${ }^{19,20}$ Thus, sofosbuvir has a high barrier to resistance. Sofosbuvir was well tolerated in phase II and III clinical trials that included several thousand patients. ${ }^{19,20}$ It is not metabolized by CYP450. Thus, sofosbuvir has few drug-drug interactions, except with potent P-glycoprotein and/or breast cancer resistance protein inducers.

\section{Drugs in Clinical Development}

\section{NS3-4A Protease Inhibitors}

NS3-4A protease inhibitors in development include secondwave, first-generation drugs that share simeprevir's properties, including broad genotypic coverage that excludes genotype 3 and a low barrier to resistance with cross-resistance with other first-generation inhibitors. The most frequently selected substitutions conferring resistance to first-generation NS3-4A protease inhibitors have been described at amino acid positions V36, T54, R155, A156, D168, and V170. ${ }^{16}$ The Q80K substitution is preferentially selected by simeprevir. Drug-drug interactions have been reported for some of them.

Compounds in phase II or III clinical development include faldaprevir (Boehringer-Ingelheim, Ingelheim, Germany), likely approved in 2014 in combination with PegIFN- $\alpha$ and ribavirin; asunaprevir (Bristol-Myers Squibb, New York, NY), 
possibly approved in 2014 or 2015; ABT-450 (Abbvie, North Chicago, IL) boosted by ritonavir, likely approved in 2014 or 2015 as part of the first all-oral, IFN-free combination for HCV genotype 1 infection; vedroprevir (Gilead, Foster City, CA); IDX-320 (Idenix Pharmaceuticals, Cambridge, MA); sovaprevir (Achillion Pharmaceuticals, New Haven, CT ), on clinical hold due to elevated alanine aminotransferase levels in a drug-drug interaction study with atazanavir; danoprevir (Hoffmann-La Roche, Basel, Switzerland), boosted by ritonavir; vaniprevir (Merck, White House Station, NJ), that will be developed in Japan only ( - Fig. 2). Second-generation NS3-4A protease inhibitors have pan genotypic antiviral activity, including genotype 3 , and a higher barrier to resistance than first-generation drugs. However, resistant HCV variants can be selected by these compounds. They include MK-5172 (Merck), possibly approved in 2015 or 2016, and ACH-2684 (Achillion) (-Fig. 2).

Nucleoside/Nucleotide Analogue Inhibitors of HCV RdRp Few nucleoside/nucleotide analogues remain at the clinical developmental stage after several programs were halted due to serious, sometimes fatal toxicity. VX-135 (Vertex Pharmaceuticals, Cambridge, MA) is a pyrimidine nucleotide analogue on partial clinical hold following the observation of reversible elevated liver enzymes in patients receiving a high dose of the drug. Mericitabine (Roche) is a modestly potent cytidine nucleoside analogue still in development (-Fig. 2).

\section{Nonnucleoside Inhibitors of HCV RdRp}

Nonnucleoside inhibitors of HCV RdRp bind to one of four allosteric sites on the polymerase, thereby altering its catalytic function. Two sites are located in the "thumb" domain and two in the "palm" domain. ${ }^{34}$ Nonnucleoside inhibitors of HCV RdRp are generally active against HCV genotype 1 only and they have a low barrier to resistance, with extensive cross resistance between drugs targeting the same allosteric site and possible cross resistance between drugs targeting different sites. Thumb-1 inhibitors include deleobuvir (BoehringerIngelheim), the development of which has been stopped in January 2014, BMS-791325 (Bristol-Myers Squibb), and TMC647055 (Janssen). The two latter belong to triple-combination regimens that will seek approval in 2015 or 2016. Thumb-2 inhibitors include filibuvir (Pfizer, New York, NY), the development of which has been stopped, lomibuvir (Vertex) and GS9669 (Gilead). Palm-1 inhibitors include setrobuvir (Roche), and ABT-333 (Abbvie), which will likely be approved in 2014 or 2015 in combination with other DAAs, and ABT-072 (Abbvie). Palm-2 inhibitors include tegobuvir (Gilead), the development of which has been stopped (-Fig. 2).

\section{NS5A Inhibitors}

NS5A inhibitors bind to domain 1 of the NS5A protein and block both replication and viral assembly and release. ${ }^{35,36}$ Due to their double mode of action, NS5A inhibitors induce a rapid and profound HCV RNA decline upon administration. First-

\section{HCV DAAS and HTAs in clinical development (January 2014)}

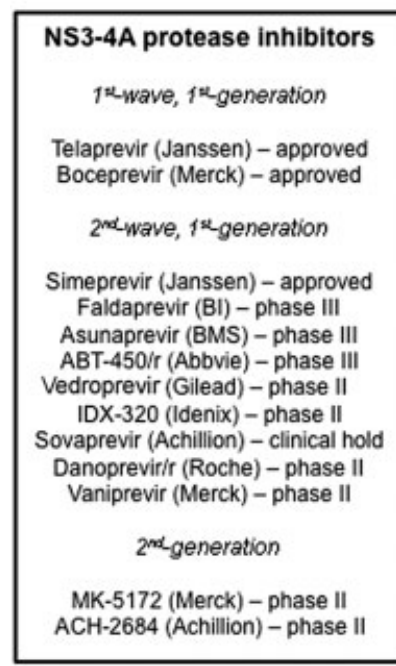

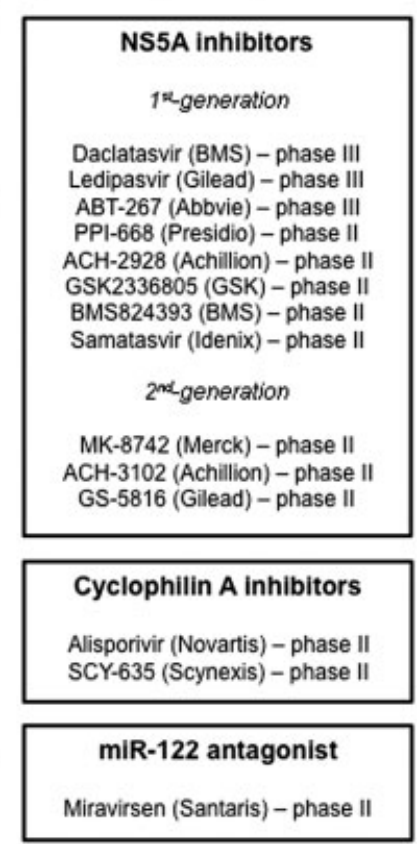

Fig. 2 Hepatitis C virus (HCV) direct-acting antivirals (DAAs) and host-targeted agents (HTAs) in clinical development in January 2014. First-generation NS3-4A protease inhibitors are active against HCV genotypes 1,2 , and sometimes 4,5 , and 6 , but not against genotype 3 , and they have a low barrier to resistance. Firstwave, first-generation drugs were approved in 2011 and are poorly tolerated, whereas second-wave inhibitors that will reach the market in 2014 and onwards are better tolerated. Second-generation NS3-4A protease inhibitors have pan genotypic activity and a higher barrier to resistance than first-generation drugs. Nucleoside/nucleotide analogue inhibitors of HCV RdRp have pangenotypic antiviral activity and a high barrier to resistance. Thumb-1, thumb-2, and palm-1 nonnucleoside inhibitors target different allosteric sites of the right-hand-shaped HCV RdRp. First-generation NS5A inhibitors are not active against all HCV genotypes and they have a low barrier to resistance. Second-generation NS5A inhibitors have pan genotypic antiviral activity and they have an improved barrier to resistance. Host-targeted agents, including cyclophilin A inhibitors and the miR-122 antagonist, have pangenotypic antiviral activity and a high barrier to resistance. r, ritonavir-boosted. 
generation NS5A inhibitors are active against genotypes 1 and 4. Some of them, but not all, are also active against genotypes 2 and/or 3. ${ }^{37}$ Their activity against genotypes 5 and 6 has not been tested in most cases. They have a low barrier to resistance, especially for genotype $1 \mathrm{a}$ and for genotype 3 for those active against this genotype. The most frequently selected substitutions are at positions M28, Q/A30, L31, and Y93 in the NS5A sequence. ${ }^{37}$ Variants bearing these substitutions persist after treatment failure in most cases, raising the issue of retreatment with a combination that includes an NS5A inhibitor.

First-generation NS5A inhibitors in development include daclatasvir (Bristol-Myers Squibb), likely approved in 2014 or 2015 for use in IFN-containing and IFN-free combinations; ledipasvir (Gilead), available as a fixed-dose combination (one pill) with sofosbuvir, likely approved in 2015; ABT267 (Abbvie), likely approved in 2014 or 2015 in combination with ritonavir-boosted ABT-450 and ABT-333; PPI-668 (Presidio Pharmaceuticals, San Francisco, CA); ACH-2928 (Achillion); GSK2336805 (GlaxoSmithKline); BMS824393 (BristolMyers Squibb); and samatasvir (Idenix) (-Fig. 2). All of them are currently being tested as part of double- or triplecombination IFN-free regimens.

Second-generation NS5A inhibitors have reached clinical development. They have pangenotypic activity and their barrier to resistance has been improved. However, they have been shown to select amino acid substitutions that confer resistance to first-generation compounds. They include MK-8742 (Merck), likely approved in 2015 or 2016 in combination with MK-5172; ACH-3102 (Achillion); and GS-5816 (Gilead), which may ultimately replace ledipasvir in the fixed-dose combination with sofosbuvir (-Fig. 2).

\section{Host-Targeted Agents}

Two classes of host-targeted agents have reached clinical development. They include specific inhibitors of cyclophilin A peptidyl-prolyl cis/trans isomerase activity and antagonists of miR-122. These compounds have pangenotypic activity, a high barrier to resistance and they are well tolerated in the absence of IFN- $\alpha$ coadministration. ${ }^{38}$ Cyclophilin inhibitors in clinical development include alisporivir (Novartis, Basel, Switzerland) and SCY-635 (Scynexis, Inc., Durham, NC) (-Fig. 2). Alisporivir was put on clinical hold due to a fatal case of acute pancreatitis that occurred in combination with PegIFN- $\alpha$ and ribavirin. It is now back in development in alloral, IFN-free combination regimens. The miR-122 antagonist miravirsen (Santaris Pharma, Copenhagen, Denmark) is available in an injectable form. Two weeks of administration reduced HCV replication by several logs. ${ }^{14}$ However, concerns have been raised as to the long-term hepatic effects of inhibiting miR-122 and the risk of steatohepatitis, fibrosis, and hepatocellular carcinoma.

\section{HCV Treatment Strategies}

\section{Until 2014}

For the past 15 years, the treatment of chronic hepatitis $C$ has been based on the use of PegIFN- $\alpha$ and ribavirin. In 2011, telaprevir and boceprevir were approved in combination with
PegIFN- $\alpha$ and ribavirin for patients infected with HCV genotype 1 . This triple combination improved the SVR rates by 15 to $20 \%$ compared with PegIFN- $\alpha$ and ribavirin alone. ${ }^{25,27-29}$ Easy-to-cure patients- patients with mild liver diseasewere those with the highest SVR rates. However, frequent and often serious side effects were observed with this regimen, especially in patients with advanced liver disease who marginally benefited from the addition of the protease inhibitor. ${ }^{26}$ Because of the complexity of these therapies and of the arrival of new, more efficient, and better-tolerated treatments, most patients who could wait for new treatment regimens were not treated in 2013 in the United States and Europe.

\section{4-2015}

Two HCV DAAs were approved in December 2013 in the United States and in the first half of 2014 in Europe: simeprevir and sofosbuvir. Four treatment options are available in 2014 for the treatment of chronic hepatitis C, including IFNcontaining and IFN-free regimens. IFN-containing options include the triple combination of PegIFN- $\alpha$, ribavirin, and simeprevir 24 to 48 weeks for patients infected with HCV genotype 1 (excluding those infected with subtype 1a with a detectable Q80K substitution in the NS3-4A protease sequence at baseline), that yields SVR rates of the order of $80 \%^{30-32}$; and the triple combination of PegIFN- $\alpha$, ribavirin, and sofosbuvir for 12 weeks for patients infected with HCV genotypes 1 to 6 that yields SVR rates of the order of $90 \%$ or more. ${ }^{20}$ IFN-free options include the combination of sofosbuvir and ribavirin 12 weeks for patients infected with HCV genotype 2, that yields SVR rates over 95\% (except in patients with cirrhosis who may need slightly longer treatment $)^{19,20}$; the combination of sofosbuvir and ribavirin 24 weeks for patients infected with HCV genotype 3, that yields SVR rates of the order of $85 \%$ in noncirrhotic patients, but appears to be suboptimal ( $\sim 60 \%$ ) in patients with cirrhosis. ${ }^{19,20}$ The combination of sofosbuvir and simeprevir, with or without ribavirin, can also be used in patients infected with $\mathrm{HCV}$ genotypes 1 and 4, based on results of a small-scale phase II trial including null responders to a prior course of treatment with extensive fibrosis or cirrhosis showing SVR rates of 95 to $100 \% .^{39}$

\section{5 and Onward}

IFN-containing regimens will progressively disappear, replaced by all-oral IFN-free regimens. Phase II trials of different drug combinations have shown SVR rates of the order of more than $90 \%$. The recent release of preliminary results from phase III trials confirmed that most, if not all patients can achieve an SVR with these regimens, at least in clinical trials. The combination of the second-wave, firstgeneration protease inhibitor ABT-450 boosted by ritonavir, the NS5A inhibitor ABT-267, and the nonnucleoside inhibitor of HCV RdRp ABT-333 plus ribavirin for 12 weeks yielded SVR rates of $95 \%$ in subtype $1 \mathrm{a}$ and $98 \%$ in subtype $1 \mathrm{~b}$ treatmentnaïve patients, and $96 \%$ in subtype $1 \mathrm{a}$ and $97 \%$ in subtype $1 \mathrm{~b}$ treatment-experienced patients (SAPPHIRE-1 and SAPPHIRE2 phase III trials). ${ }^{40,41}$ In the ION-1, ION-2, and ION-3 phase III 
trials with the fixed-dose combination of sofosbuvir and ledipasvir in patients infected with HCV genotype 1, the SVR rates were $97.7 \%$ and $97.2 \%$ with or without ribavirin, respectively, after 12 weeks of therapy in treatment-naïve patients (including 16\% with cirrhosis); 94.0\%, 93.1\%, and $95.4 \%$ without ribavirin for 8 weeks, with ribavirin for 8 weeks and without ribavirin for 12 weeks in treatment-naïve patients, respectively; $93.6 \%$ and $96.4 \%$ with or without ribavirin, respectively, after 12 weeks, and $99.1 \%$ and $99.1 \%$ with or without ribavirin, respectively, after 24 weeks of therapy in treatment-experienced patients (including $20 \%$ with cirrhosis). ${ }^{42}$ Other phase III trials are ongoing with the same and other drug combinations.

\section{Conclusion}

Curing HCV infection is an easy task, provided that appropriate tools-potent drug combinations with a high barrier to resistance-are used. This has become possible with the discovery of several new HCV DAAs and HTAs that have reached phase II and phase III clinical development. SVR rates as high as $95 \%$ or more have been reported in phase III trials with such combinations, with favorable safety profiles and limited drug-drug interactions. These results, together with the dozens of ongoing studies with different treatment regimens in various populations of $\mathrm{HCV}$-infected patients, indicate that the IFN era is getting to its end in HCV therapy. New all-oral, IFN-free strategies, described in detail in various articles in this issue, will take over. However, they will raise new issues that will need to be tackled, including the need for broad-scale screening, access to care, and the high costs associated with the drugs.

\section{Conflict of Interest Disclosure}

The author has received research grants from Gilead Sciences. He has served as an advisor for Abbott, Abbvie, Achillion, Boehringer-Ingelheim, Bristol-Myers Squibb, Gilead Sciences, Idenix, Janssen, Merck, Novartis, and Roche.

\section{References}

1 Choo QL, Kuo G, Weiner AJ, Overby LR, Bradley DW, Houghton M. Isolation of a cDNA clone derived from a blood-borne non-A, nonB viral hepatitis genome. Science 1989;244(4902):359-362

2 Hoofnagle JH, Mullen KD, Jones DB, et al. Treatment of chronic non$A$, non-B hepatitis with recombinant human alpha interferon. A preliminary report. N Engl J Med 1986;315(25):1575-1578

3 Reichard O, Andersson J, Schvarcz R, Weiland O. Ribavirin treatment for chronic hepatitis C. Lancet 1991;337(8749):1058-1061

4 Pawlotsky JM, Dahari H, Neumann AU, et al. Antiviral action of ribavirin in chronic hepatitis C. Gastroenterology 2004;126(3): 703-714

5 Fried MW, Shiffman ML, Reddy KR, et al. Peginterferon alfa-2a plus ribavirin for chronic hepatitis C virus infection. N Engl J Med 2002; 347(13):975-982

6 Manns MP, McHutchison JG, Gordon SC, et al. Peginterferon alfa-2b plus ribavirin compared with interferon alfa- $2 \mathrm{~b}$ plus ribavirin for initial treatment of chronic hepatitis C: a randomised trial. Lancet 2001;358(9286):958-965
7 Pawlotsky JM. Treatment of chronic hepatitis C: current and future. Curr Top Microbiol Immunol 2013;369:321-342

8 Lanford RE, Guerra B, Lee H, et al. Antiviral effect and virus-host interactions in response to alpha interferon, gamma interferon, poly(i)-poly(c), tumor necrosis factor alpha, and ribavirin in hepatitis C virus subgenomic replicons. J Virol 2003;77(2): 1092-1104

9 Ge D, Fellay J, Thompson AJ, et al. Genetic variation in IL28B predicts hepatitis $C$ treatment-induced viral clearance. Nature 2009;461(7262):399-401

10 Neumann AU, Lam NP, Dahari H, et al. Hepatitis C viral dynamics in vivo and the antiviral efficacy of interferon-alpha therapy. Science 1998;282(5386):103-107

11 Dahari H, Guedj J, Perelson AS, Layden TJ. Hepatitis C viral kinetics in the era of direct acting antiviral agents and IL28B. Curr Hepat Rep 2011;10(3):214-227

12 Rupp D, Bartenshlager R. Targets for antiviral therapy of hepatitis C. Semin Liver Dis 2014;34:9-21

13 Flisiak R, Horban A, Gallay P, et al. The cyclophilin inhibitor Debio025 shows potent anti-hepatitis $C$ effect in patients coinfected with hepatitis $C$ and human immunodeficiency virus. Hepatology 2008;47(3):817-826

14 Janssen HL, Reesink HW, Lawitz EJ, et al. Treatment of HCV infection by targeting microRNA. N Engl J Med 2013;368(18): 1685-1694

15 Delang L, Vliegen I, Leyssen P, Neyts J. In vitro selection and characterization of HCV replicons resistant to multiple non-nucleoside polymerase inhibitors. J Hepatol 2012;56(1):41-48

16 Pawlotsky JM. Treatment failure and resistance with direct-acting antiviral drugs against hepatitis C virus. Hepatology 2011;53(5): 1742-1751

17 Chu TW, Kulkarni R, Gane EJ, et al. Effect of IL28B genotype on early viral kinetics during interferon-free treatment of patients with chronic hepatitis C. Gastroenterology 2012;142(4):790-795

18 Zeuzem S, Soriano V, Asselah T, et al. Faldaprevir and deleobuvir for HCV genotype 1 infection. N Engl J Med 2013;369(7):630-639

19 Jacobson IM, Gordon SC, Kowdley KV, et al; POSITRON Study; FUSION Study. Sofosbuvir for hepatitis C genotype 2 or 3 in patients without treatment options. N Engl J Med 2013;368(20): 1867-1877

20 Lawitz E, Mangia A, Wyles D, et al. Sofosbuvir for previously untreated chronic hepatitis C infection. N Engl J Med 2013; 368(20):1878-1887

21 Zeuzem S, Dusheiko GM, Salupere R, et al. Sofosbuvir + ribavirin for 12 or 24 weeks for patients with HCV genotype 2 or 3: the VALENCE trial. Hepatology 2013;58(Suppl 1):733A

22 Guedj J, Perelson AS. Second-phase hepatitis C virus RNA decline during telaprevir-based therapy increases with drug effectiveness: implications for treatment duration. Hepatology 2011;53(6): 1801-1808

23 Thomas E, Ghany MG, Liang TJ. The application and mechanism of action of ribavirin in therapy of hepatitis $\mathrm{C}$. Antivir Chem Chemother 2012;23(1):1-12

24 Back D, Else L. The importance of drug-drug interactions in the DAA era. Dig Liver Dis 2013;45(Suppl 5):S343-S348

25 Bacon BR, Gordon SC, Lawitz E, et al; HCV RESPOND-2 Investigators. Boceprevir for previously treated chronic HCV genotype 1 infection. N Engl J Med 2011;364(13):1207-1217

26 Hézode C, Fontaine H, Dorival C, et al; CUPIC Study Group. Triple therapy in treatment-experienced patients with HCV-cirrhosis in a multicentre cohort of the French Early Access Programme (ANRS CO20-CUPIC) - NCT01514890. J Hepatol 2013;59(3):434-441

27 Jacobson IM, McHutchison JG, Dusheiko G, et al; ADVANCE Study Team. Telaprevir for previously untreated chronic hepatitis $C$ virus infection. N Engl J Med 2011;364(25):2405-2416

28 McHutchison JG, Manns MP, Muir AJ, et al; PROVE3 Study Team. Telaprevir for previously treated chronic HCV infection. N Engl J Med 2010;362(14):1292-1303 
29 Poordad F, McCone J Jr, Bacon BR, et al; SPRINT-2 Investigators. Boceprevir for untreated chronic HCV genotype 1 infection. N Engl J Med 2011;364(13):1195-1206

30 Jacobson I, Dore GJ, Foster GR, et al. Simeprevir (TMC435) with peginterferon/ribavirin for chronic HCV genotype-1 infection in treatment-naive patients: results from QUEST-1, a Phase III trial. J Hepatol 2013;58(Suppl 1):S574

31 Manns M, Marcellin P, Poordad FPF, et al. Simeprevir (TMC435) with peginterferon/ribavirin for treatment of chronic HCV genotype-1 infection in treatment-naive patients: results from QUEST2, a Phase III trial. J Hepatol 2013;58(Suppl 1):S568

32 Poordad F, Manns MP, Marcellin P, et al. Simeprevir (TMC435) with peginterferon/ribavirin for treatment of chronic HCV genotype-1 infection in treatment-naive patients: results from QUEST-2, a Phase III trial. Gastroenterology 2013;144:S151-S151

33 Lam AM, Espiritu C, Bansal S, et al. Genotype and subtype profiling of PSI-7977 as a nucleotide inhibitor of hepatitis C virus. Antimicrob Agents Chemother 2012;56(6):3359-3368

34 Haudecoeur R, Peuchmaur M, Ahmed-Belkacem A, Pawlotsky JM, Boumendjel A. Structure-activity relationships in the development of allosteric hepatitis C virus RNA-dependent RNA polymerase inhibitors: ten years of research. Med Res Rev 2013;33(5):934-984

35 Guedj J, Dahari H, Rong L, et al. Modeling shows that the NS5A inhibitor daclatasvir has two modes of action and yields a shorter estimate of the hepatitis C virus half-life. Proc Natl Acad Sci U S A 2013;110(10):3991-3996

36 McGivern DR, Masaki T, Ping LF, et al. Kinetic analyses of antiviral suppression by NS5A inhibitors reveal early and potent inhibition of viral assembly and release of infectious virus. Hepatology 2013; 58(Suppl 1):246A

37 Pawlotsky JM. NS5A inhibitors in the treatment of hepatitis C. J Hepatol 2013;59(2):375-382
38 Pawlotsky JM, Sarin SK, Foster GR, et al. Alisporivir plus ribavirin achieves high rates of sustained HCV clearance (SVR24) as interferon (IFN)-free or IFN-add-on regimen in treatment-naive patients with HCV GT2 or GT3: final results from VITAL-1 study. Hepatology 2012;56:309A-310A

39 Jacobson IM, Ghalib RH, Rodriguez-Torres M, et al. SVR results of a once-daily regimen of simeprevir (TMC435) plus sofosbuvir (GS-7977) with or without ribavirin in cirrhotic and noncirrhotic HCV genotype 1 treatment-naïve and prior null responder patients: the COSMOS study. Hepatology 2013;58 (Suppl 1):1379A

40 Abbvie. Abbvie releases first of six phase III results from investigational all-oral, interferon-free, 12-week regimen, showing 96 percent SVR12 in genotype 1 hepatitis C patients new to therapy. Available at: http://abbvie.mediaroom.com/2013-11-18-AbbVieReleases-First-of-Six-Phase-III-Results-from-Investigational-AllOral-Interferon-Free-12-week-Regimen-Showing-96-PercentSVR12-in-Genotype-1-Hepatitis-C-Patients-New-to-Therapy. Accessed February 1, 2014

41 Abbvie. Abbvie demonstrates 96 percent SVR-12 in its phase III study of treatment experienced patients with genotype 1 hepatitis C. Available at: http://abbvie.mediaroom.com/2013-12-10-AbbVie-Demonstrates-96-percent-SVR-12-in-its-Phase-III-Study-ofTreatment-Experienced-Patients-with-Genotype-1-Hepatitis-C

42 Gilead. Gilead announces SVR12 rates from three phase 3 studies evaluating a once daily fixed dose-combination-of-sofosbuvirand-ledipasvir-for-genotype-1-hepatitis $\mathrm{C}$ patients. Available at: http://www.gilead.com/news/press-releases/2013/12/gileadannounces-svr12-rates-from-three-phase-3-studies-evaluating-aoncedaily-fixeddose-combination-of-sofosbuvir-and-ledipasvirfor-genotype-1-hepatitis-c-patients?mode=print. Accessed February 1,2014 